Gut and Liver, Vol. 12, No. 4, July 2018, pp. 420-425

\title{
An Elevated Platelet Count Increases the Risk of Relapse in Ulcerative Colitis Patients with Mucosal Healing
}

\author{
Asuka Nakarai ${ }^{1}$, Jun Kato ${ }^{2}$, Sakiko Hiraoka ${ }^{1}$, Shiho Takashima ${ }^{1}$, Toshihiro Inokuchi ${ }^{1}$, Masahiro Takahara ${ }^{1}$, \\ Yuusaku Sugihara ${ }^{1}$, Keita Harada ${ }^{3}$, and Hiroyuki Okada ${ }^{1}$ \\ ${ }^{1}$ Department of Gastroenterology and Hepatology, Okayama University Graduate School of Medicine, Dentistry and Pharmaceutical Sciences, \\ Okayama, ${ }^{2}$ Second Department of Internal Medicine, Wakayama Medical University, Wakayama, and ${ }^{3}$ Department of Endoscopy, Okayama \\ University Graduate School of Medicine, Dentistry and Pharmaceutical Sciences, Okayama, Japan
}

Background/Aims: Although mucosal healing $(\mathrm{MH})$ has been considered a treatment goal for patients with ulcerative colitis (UC), the risk factors predictive of relapse in patients who achieve $\mathrm{MH}$ are unknown. Because the platelet count has been shown to be a marker of inflammation in inflammatory bowel diseases, this study aimed to assess whether the platelet count could predict relapse in UC patients with MH. Methods: A prospective observational study was performed. UC patients with $\mathrm{MH}$ were consecutively enrolled in the study and monitored for at least 2 years or until relapse. The correlation between the incidence of relapse and the platelet count at the time of study enrollment was examined. Results: In total, 43 patients were enrolled, and 14 patients (33\%) relapsed. The median platelet count at the time of enrollment in the patients who relapsed significantly differed from that in the patients who did not relapse $\left(27.2 \times 10^{4} /\right.$ $\mu \mathrm{L}$ vs $23.8 \times 10^{4} / \mu \mathrm{L}$, respectively; $p=0.016$ ). A platelet count $>25.0 \times 10^{4} / \mu \mathrm{L}$ was a significant risk factor for relapse based on a multivariate analysis (hazard ratio, 4.85; 95\% confidence interval, 1.07 to 25.28), and according to the KaplanMeier analysis, this cutoff could identify patients susceptible to relapse ( $p=0.041$, log-rank test). Conclusions: The platelet count could be used as a predictor of relapse in UC patients with MH. (Gut Liver 2018;12:420-425)

Key Words: Colitis, ulcerative; Mucosal healing; Platelet count; Recurrence

\section{INTRODUCTION}

Ulcerative colitis (UC) is an idiopathic chronic inflammatory disorder that affects the innermost lining or mucosa of the colon and rectum, and UC patients have symptoms such as diarrhea and bloody stool. The clinical course of UC is characterized by repetitive cycles of relapses and remissions despite patients receiving appropriate treatment. Therefore, predictors of clinical relapse in UC are desirable.

Mucosal healing $(\mathrm{MH})$ has recently been recognized as a treatment goal of UC because patients with $\mathrm{MH}$ have been shown to have a reduced risk of relapse. ${ }^{1}$ Thus, achieving MH is a mandatory requirement in clinically treating UC. Previously, biomarkers that predict $\mathrm{MH}$ have extensively been explored, and several blood, fecal, and biological biomarkers for $\mathrm{MH}$ have been established, including fecal calprotectin, fecal immunochemical test, C-reactive protein (CRP), and erythrocyte sedimentation rate. ${ }^{2-7}$ However, reports regarding biomarkers associated with the prognosis of inflammatory bowel disease (IBD) patients who achieved MH have been scarce.

We previously reported that platelet count could reflect endoscopic inflammation in individuals with quiescent UC and predict a slight endoscopic relapse, a shift in the Mayo endoscopic subscore (MES) from 0 to $1 .^{8}$ Therefore, we hypothesized that platelet count could predict the risk of relapse in UC patients with $\mathrm{MH}$, particularly when $\mathrm{MH}$ was strictly defined (e.g., MES 0 throughout the colorectum).

The aim of this study was to assess whether platelet count could predict relapse in UC patients who achieved MH.

\footnotetext{
Correspondence to: Sakiko Hiraoka

Department of Gastroenterology and Hepatology, Okayama University Graduate School of Medicine, Dentistry and Pharmaceutical Sciences, 2-5-1 Shikata-cho, Kita-ku, Okayama 700-8558, Japan

Tel: +81-86-235-7219, Fax: +81-86-225-5991, E-mail: sakikoh@cc.okayama-u.ac.jp

Received on June 1, 2017. Revised on September 8, 2017. Accepted on September 22, 2017.

pISSN 1976-2283 eISSN 2005-1212 https://doi.org/10.5009/gnl17236

@ This is an Open Access article distributed under the terms of the Creative Commons Attribution Non-Commercial License (http://creativecommons.org/licenses/by-nc/4.0) which permits unrestricted non-commercial use, distribution, and reproduction in any medium, provided the original work is properly cited.
} 


\section{MATERIALS AND METHODS}

\section{Patients}

A prospective observational study was performed. Between January 2014 and January 2015, UC patients in clinical remission for 3 months or more and showed MH based on colonoscopy were consecutively enrolled. A diagnosis of UC was based on typical endoscopic and histological assessments. Clinical disease activity was evaluated using the Mayo score (scoring systems for assessment of UC), and clinical remission was defined as a Mayo stool frequency subscore of either 0 or 1 and a Mayo rectal bleeding subscore of $0 .{ }^{9}$ In this study, $\mathrm{MH}$ was defined as an MES 0 alone throughout the colorectum.

Blood tests including serum albumin and CRP as well as a full blood count for each patient were evaluated using routine assays at the time of enrolment. Patients were followed for at least 2 years after enrolment or until relapse with approximately bimonthly visit intervals. Blood tests were performed at regular visits during the follow-up period if necessary. Changes in medications during sustained clinical remission (e.g., tapering of corticosteroids and tacrolimus) were made at the discretion of each attending physician. The primary endpoint was the incidence of clinical relapse during the follow-up period according to the measured platelet count at enrolment. Clinical relapse was defined as an addition or modification of medications due to worsening symptoms. This study was approved by the Institutional Review Board at Okayama University Graduate School of Medicine. Informed consent was obtained from each patient. There were no conflicts of interest or sponsors in this study.

\section{Statistical analysis}

Statistical analysis was conducted using the JMP program version 9 (SAS Institute, Cary, NC, USA). Values were expressed as the median and interquartile range (IQR). The Mann-Whitney test was used to compare blood parameters between patients with and without relapse. Receiver operating characteristic curves were constructed to define a cutoff level for each parameter. The Cox proportional hazards regression model was used to calculate hazard ratios (HRs) with 95\% confidence intervals (CIs) for the risk of relapse. The cumulative percentage of relapse-free patients was determined using Kaplan-Meier analysis and com- pared using the log-rank test. A power analysis was performed using a PS power and sample size calculator (http://biostat. mc.vanderbilt.edu/PowerSampleSize). We made our calculations based on the detection of a 3-fold difference in the probability of relative risk of clinical relapse between the two groups with 80\% power and 0.05 probability level. All p-values were twosided and considered significant when they were less than 0.05 .

\section{RESULTS}

A total of 43 patients in clinical remission with MH (MES 0 throughout the colorectum) were enrolled in this study. Patient

Table 1. Patient Demographics and Clinicopathological Characteristics

\begin{tabular}{lc}
\hline \multicolumn{1}{c}{ Variable } & Value \\
\hline Total no. & 43 \\
Male sex & $20(47)$ \\
Age, yr & $47(35-61)$ \\
Extent of disease & \\
Pancolitis & $30(70)$ \\
Left-side colitis & $9(21)$ \\
Proctitis & $4(9)$ \\
Age at onset, yr & $34(19-43)$ \\
Duration of disease, yr & $9(5-17)$ \\
Medications & \\
Aminosalicylate & $37(86)$ \\
Corticosteroids & $4(9)$ \\
Mercaptopurine/azathioprine & $15(35)$ \\
Tacrolimus & $1(2)$ \\
Biologics & $1(2)$ \\
Laboratory parameter & \\
CRP, mg/L & $0.4(0.2-0.8)$ \\
WBC, $/ \mu \mathrm{L}$ & $5,450(4,070-6,630)$ \\
Hemoglobin, g/dL & $13.9(12.3-14.9)$ \\
Platelet count, $\times 10^{4} / \mu \mathrm{L}$ & $24.2(22.3-27.7)$ \\
Albumin, g/dL & $4.4(4.2-4.6)$ \\
\hline
\end{tabular}

Data are presented as number (\%) or median (interquartile range). CRP, C-reactive protein; WBC, leukocyte count.

Table 2. Laboratory Parameters in Patients with and without Relapse

\begin{tabular}{lccc}
\hline \multicolumn{1}{c}{ Laboratory parameter } & Relapse $(\mathrm{n}=14)$ & No relapse $(\mathrm{n}=29)$ & $\mathrm{p}$-value \\
\hline $\mathrm{CRP}, \mathrm{mg} / \mathrm{L}$ & $0.7(0.2-1.3)$ & $0.3(0.2-0.7)$ & 0.318 \\
$\mathrm{WBC}, / \mu \mathrm{L}$ & $5,510(4,859-7,200)$ & $5,230(4,050-6,460)$ & 0.468 \\
Hemoglobin, $\mathrm{g} / \mathrm{dL}$ & $13.2(12.3-14.7)$ & $14.4(12.3-15.0)$ & 0.276 \\
Platelet count, $\times 10^{4} / \mu \mathrm{L}$ & $27.2(23.6-30.1)$ & $23.8(21.9-25.9)$ & 0.016 \\
Albumin, $\mathrm{g} / \mathrm{dL}$ & $4.4(4.2-4.5)$ & $4.4(4.2-4.6)$ & 0.764 \\
\hline
\end{tabular}

Data are presented as median (interquartile range).

CRP, C-reactive protein; WBC, leukocyte count. 
characteristics and blood parameters at the time of enrolment are shown in Table 1 . The median observation period was 29 months (IQR, 21 to 31 months), and 14 patients (33\%) relapsed.

The values of the blood parameters at enrollment were compared between patients with and without relapse (Table 2). Platelet count alone showed statistical significance and was elevated in patients who relapsed than in those who did not re-

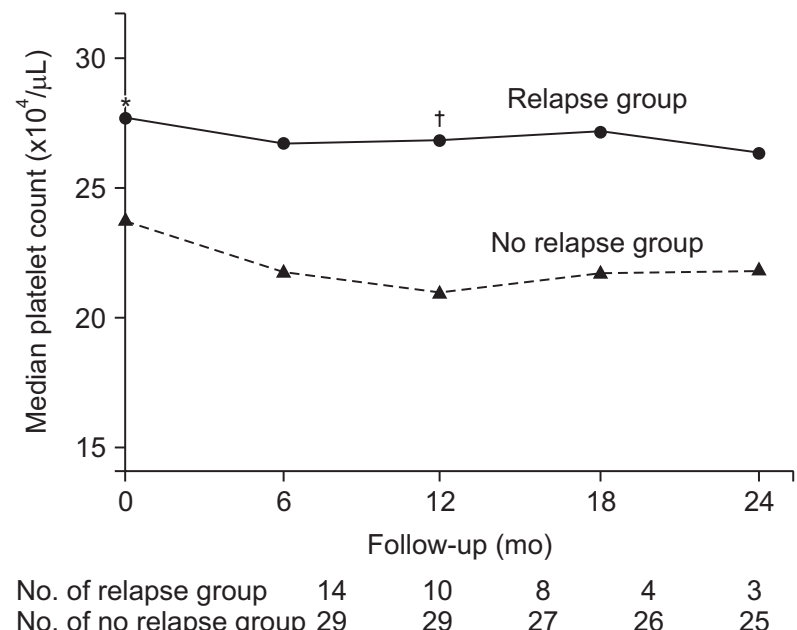

Fig. 1. Differences in platelet counts during the follow-up period between patients with and without relapse. The platelet count was compared at the time of study enrollment and at 6-month intervals during the follow-up period. The platelet counts at the time of study enrollment and 1 year after enrollment significantly differed.

${ }^{*} 27.2 \times 10^{4} / \mu \mathrm{L}$ vs $23.8 \times 10^{4} / \mu \mathrm{L}, \mathrm{p}=0.016 ;{ }^{\dagger} 26.5 \times 10^{4} / \mu \mathrm{L}$ vs $21.3 \times 10^{4} / \mu \mathrm{L}$, $\mathrm{p}=0.004$. lapse $\left(27.2 \times 10^{4} / \mu \mathrm{L}\right.$ vs $23.8 \times 10^{4} / \mu \mathrm{L}$, respectively, $\left.\mathrm{p}=0.016\right)$. Platelet count was constantly higher during the follow-up period in patients who relapsed (Fig. 1). The receiver operating characteristic curve analysis revealed that $25 \times 10^{4} / \mu \mathrm{L}$ was an optimal cutoff of platelet count for discriminating between patients with and without relapse, and clinical characteristics did not differ significantly between patients above and below the cutoff (data not shown).

The multivariate Cox proportional hazards regression analysis revealed that a platelet count $>25 \times 10^{4} / \mu \mathrm{L}$ was an independent predictive factor of clinical relapse (HR, 4.85; 95\% CI, 1.07 to $25.28 ; \mathrm{p}=0.040$ ) (Table 3). Moreover, we performed further univariate and multivariate analyses with addition of patient clinical factors (extent of disease, duration of disease, and medications) to explanatory parameters. As a result, a platelet count $\geq 25 \times 10^{4} / \mu \mathrm{L}$ and a mercaptopurine/azathioprine use were independent predictive factors of clinical relapse (HR, 3.39; 95\% CI, 1.03 to $12.17 ; \mathrm{p}=0.045$ and HR, 3.49; 95\% CI, 1.16 to $11.00 ; p=0.027$, respectively). The Kaplan-Meier curves of the time to relapse showed that the cumulative relapse rate was significantly higher in patients with a platelet count $\geq 25 \times 10^{4} / \mu \mathrm{L}$ than those with a platelet count $<25 \times 10^{4} / \mu \mathrm{L}(\mathrm{p}=0.041$, log-rank test) (Fig. 2A). Relapse was confirmed endoscopically in 10 of 14 patients who showed clinical relapse. The cumulative endoscopic relapse rate was also significantly higher in patients with a platelet count $\geq 25 \times 10^{4} / \mu \mathrm{L}$ than those with a platelet count $<25 \times 10^{4} / \mu \mathrm{L}$ ( $\mathrm{p}=0.021$, log-rank test) (Fig. $2 \mathrm{~B}$ ).

Table 3. Cox Proportional Hazards Regression Analysis of the Risk of Relapse

\begin{tabular}{|c|c|c|c|c|}
\hline \multirow{2}{*}{ Variable } & \multicolumn{2}{|c|}{ Univariate } & \multicolumn{2}{|c|}{ Multivariate } \\
\hline & HR $(95 \%$ CI) & $\mathrm{p}$-value & HR (95\% CI) & p-value \\
\hline \multicolumn{5}{|l|}{ CRP, mg/L } \\
\hline$<0.3$ & 1 & & 1 & \\
\hline$\geq 0.3$ & $1.01(0.37-3.67)$ & 0.990 & $1.48(0.39-6.75)$ & 0.569 \\
\hline \multicolumn{5}{|l|}{$\mathrm{WBC}, / \mu \mathrm{L}$} \\
\hline$<4,700$ & 1 & & 1 & \\
\hline$\geq 4,700$ & $1.54(0.48-6.81)$ & 0.491 & $1.36(0.31-7.29)$ & 0.690 \\
\hline \multicolumn{5}{|l|}{ Hemoglobin, g/dL } \\
\hline$\leq 13.5$ & 1 & & 1 & \\
\hline$>13.5$ & $0.47(1.44-1.37)$ & 0.168 & $1.00(0.20-4.53)$ & 1.000 \\
\hline \multicolumn{5}{|l|}{ Platelet count, $\times 10^{4} / \mu \mathrm{L}$} \\
\hline$<25.0$ & 1 & & 1 & \\
\hline$\geq 25.0$ & $2.99(1.02-9.79)$ & 0.046 & $4.85(1.07-25.28)$ & 0.04 \\
\hline \multicolumn{5}{|l|}{ Albumin, g/dL } \\
\hline$\leq 4.3$ & 1 & & 1 & \\
\hline$>4.3$ & $0.89(0.30-2.61)$ & 0.830 & $1.71(0.41-7.88)$ & 0.462 \\
\hline
\end{tabular}

Adjusted for sex and age.

HR, hazard ratio; CI, confidence interval; CRP, C-reactive protein; WBC, leukocyte count. 

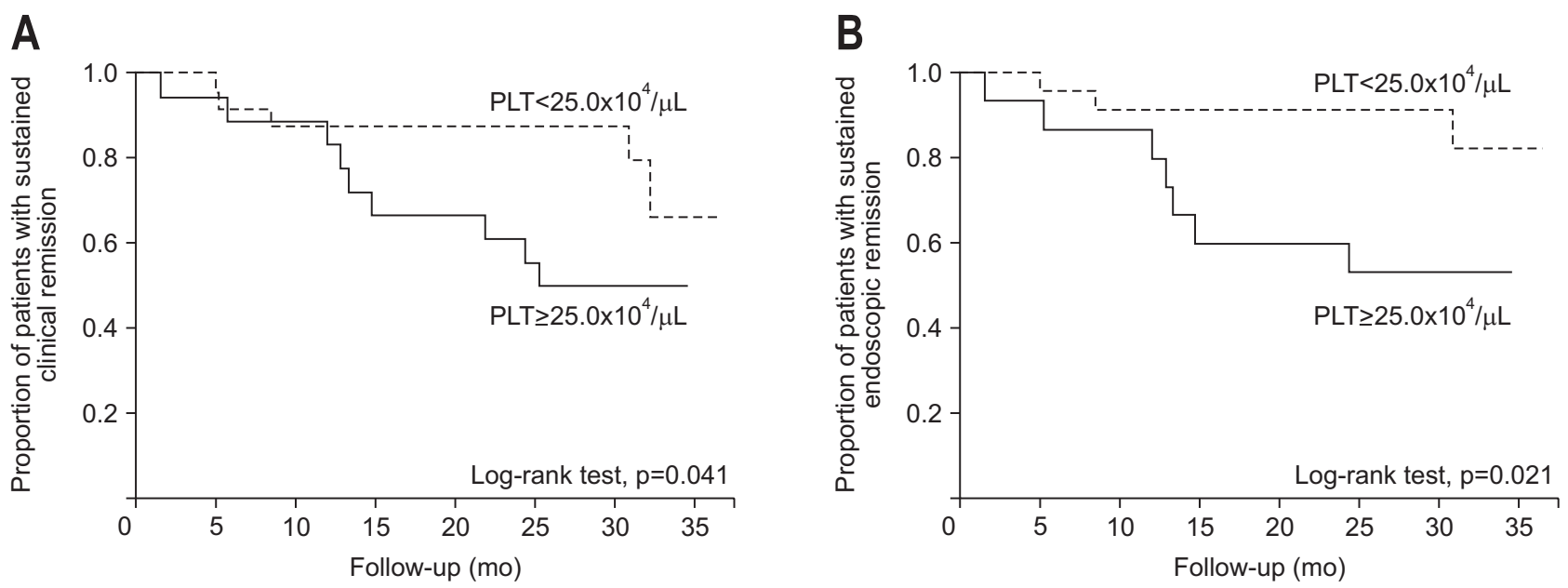

Fig. 2. Kaplan-Meier curves of time to relapse based on the platelet count (PLT) at the time of enrollment. (A) The cumulative relapse-free rate was statistically significantly different between patients with a PLT greater than or equal to and patients with a PLT less than or equal to $\geq 25 \times 10^{4} / \mu \mathrm{L}$ and those with a PLT $<25 \times 10^{4} / \mu \mathrm{L}$ ( $\mathrm{p}=0.041$, log-rank test). (B) The cumulative endoscopic relapse-free rate also significantly differed between the two groups ( $\mathrm{p}=0.021$, log-rank test).

\section{DISCUSSION}

In this study, we demonstrated that an elevated platelet count could predict relapse in clinically quiescent UC patients with MH. Recent clinical studies have underlined the importance of achieving $\mathrm{MH}$ in UC, and treatment progress for UC has made $\mathrm{MH}$ a practical treatment goal for most UC patients. However, even patients who achieved MH can relapse, and biomarkers predicting the prognosis of these patients are still lacking. Focusing on platelet count (a simple biomarker) could identify patients with $\mathrm{MH}$ expected to have a more favorable disease course.

Previous reports carried out hematological examinations on UC patients with activity or quiescence disease as well as on healthy controls, and showed that the mean platelet count was higher in patients with active disease than in either patients with inactive UC or healthy controls. ${ }^{10,11}$ In addition, we previously examined the platelet count in asymptomatic UC patients with MES 0 and revealed that the platelet count significantly increased in patients who presented a change in MES from 0 to 1 than that in patients who maintained MES $0 .{ }^{8}$ Thus, the platelet count reflects UC activity, and the worsening of endoscopic findings of patients in clinical remission who do not present any symptom changes could be recognized by an increase in the platelet count. Based on these previous findings, the current study was planned, which revealed that the platelet count in patients with $\mathrm{MH}$ is a significant predictor of relapse.

Several biological mechanisms have been presumed regarding the association between IBD activity and platelet count. Platelets are broadly activated during inflammatory responses and upon tissue injury, and activated platelets express the CD40 ligand through which they can specifically interact with many immune and non-immune cells that express CD $40 .{ }^{12,13}$ This causal cor- relation may be attributed to the fact that CD40 ligand-positive platelets are present in the circulation in several inflammatory conditions, ${ }^{14,15}$ including IBD. ${ }^{16}$ In addition, activated platelets frequently aggregate in the inflamed mucosa (especially in ulcerative lesions) and were close to regions of dense neutrophil infiltration. An increase in the number of activated platelets in colonic lesions was associated with an increase in the number of infiltrating neutrophils and was related to the severity of the disease. $^{17}$

As a predictor of the prognosis of patients with $\mathrm{MH}$, the histological findings of colonic mucosa have been suggested as a marker. According to recent studies, achievement of both endoscopic and histological remission was associated with better long-term outcomes compared with endoscopic remission alone. ${ }^{18-20}$ However, histological examinations require endoscopy with a biopsy and are therefore burdensome for both patients and physicians. In contrast, the platelet count can be measured easily and has a great advantage for use in clinical practice. In this context, recently, Mooiweer et al. ${ }^{21}$ reported that fecal calprotectin could predict relapse in IBD patients with MH more efficiently than histological findings. Future work should examine the correlations between platelet count, fecal markers and histology in UC.

In this study, relapse occurred in six of 43 patients (14\%) with $\mathrm{MH}$ within 1 year and in 14 of 43 patients (33\%) during the entire study period. In this regard, previous reports indicated $19 \%$ to $27 \%$ of the 1-year relapse rate of UC patients with MES $0 .^{22,23}$ One explanation for the lower rate of relapse in our cohort may be the relatively frequent use of immunomodulators. The platelet count appears to be sufficiently sensitive for predicting relapse because statistical significance was observed in our cohort with the lower rate of relapse. In this context, our findings should be validated in other cohorts of UC patients with MH. 
The definition of $\mathrm{MH}$ in this study was MES 0 , because recent reports suggested that UC patients with MES 1 had a higher risk of relapse than those with MES $0 .{ }^{8,22,24}$ However, because a considerable number of reports have adopted MES of either 0 or 1 as $\mathrm{MH}$, the predictive performance of platelet count should also be examined in patients with a quiescent UC and an MES of 0 or 1 .

Despite the novel findings of this study, the small cohort size and enrolment at a single institution were the major limitations of this study. The small sample size could have led to selection bias, although it was statistically calculated beforehand. In addition, the wide variety of medications taken by the patients during follow-up might cause bias. A lack of endoscopic findings at clinical relapse is also a drawback. Previous studies indicated that iron deficiency increases and iron supplementation decreases platelet count in IBD patients with anemia. ${ }^{25,26}$ Although our analysis did not include parameters associated with iron and ferritin levels, our patients did not show anemia due to $\mathrm{MH}$ and hemoglobin levels did not differ significantly between patients with and without relapse. Therefore, a lack of such parameters does not appear to yield large bias.

In conclusion, our study revealed that an elevated platelet count could predict the risk of relapse in clinically quiescent UC patients with $\mathrm{MH}$. In an era when $\mathrm{MH}$ is nothing special, our findings could greatly benefit the clinical maintenance of UC.

\section{CONFLICTS OF INTEREST}

No potential conflict of interest relevant to this article was reported.

\section{REFERENCES}

1. Peyrin-Biroulet L, Ferrante M, Magro F, et al. Results from the 2nd Scientific Workshop of the ECCO. I: Impact of mucosal healing on the course of inflammatory bowel disease. J Crohns Colitis 2011;5:477-483.

2. Lobatón T, Rodríguez-Moranta F, Lopez A, Sánchez E, RodríguezAlonso L, Guardiola J. A new rapid quantitative test for fecal calprotectin predicts endoscopic activity in ulcerative colitis. Inflamm Bowel Dis 2013;19:1034-1042.

3. Önal İK, Beyazit Y, Şener B, et al. The value of fecal calprotectin as a marker of intestinal inflammation in patients with ulcerative colitis. Turk J Gastroenterol 2012;23:509-514.

4. Nakarai A, Kato J, Hiraoka S, et al. Evaluation of mucosal healing of ulcerative colitis by a quantitative fecal immunochemical test. Am J Gastroenterol 2013;108:83-89.

5. Takashima S, Kato J, Hiraoka S, et al. Evaluation of mucosal healing in ulcerative colitis by fecal calprotectin vs. fecal immunochemical test. Am J Gastroenterol 2015;110:873-880.

6. Solem CA, Loftus EV Jr, Tremaine WJ, Harmsen WS, Zinsmeister AR, Sandborn WJ. Correlation of C-reactive protein with clinical, endoscopic, histologic, and radiographic activity in inflammatory bowel disease. Inflamm Bowel Dis 2005;11:707-712.

7. Bitton A, Peppercorn MA, Antonioli DA, et al. Clinical, biological, and histologic parameters as predictors of relapse in ulcerative colitis. Gastroenterology 2001;120:13-20.

8. Nakarai A, Kato J, Hiraoka S, et al. Prognosis of ulcerative colitis differs between patients with complete and partial mucosal healing, which can be predicted from the platelet count. World J Gastroenterol 2014;20:18367-18374.

9. Schroeder KW, Tremaine WJ, Ilstrup DM. Coated oral 5-aminosalicylic acid therapy for mildly to moderately active ulcerative colitis. A randomized study. N Engl J Med 1987;317:1625-1629.

10. Kapsoritakis AN, Koukourakis MI, Sfiridaki A, et al. Mean platelet volume: a useful marker of inflammatory bowel disease activity. Am J Gastroenterol 2001;96:776-781.

11. Kayahan H, Akarsu M, Ozcan MA, et al. Reticulated platelet levels in patients with ulcerative colitis. Int J Colorectal Dis 2007;22:1429-1435.

12. Lefer AM. Platelets: unindicted coconspirators in inflammatory tissue injury. Circ Res 2000;87:1077-1078.

13. Rizvi M, Pathak D, Freedman JE, Chakrabarti S. CD40-CD40 ligand interactions in oxidative stress, inflammation and vascular disease. Trends Mol Med 2008;14:530-538.

14. Khan SY, Kelher MR, Heal JM, et al. Soluble CD40 ligand accumulates in stored blood components, primes neutrophils through $\mathrm{CD} 40$, and is a potential cofactor in the development of transfusion-related acute lung injury. Blood 2006;108:2455-2462.

15. Phipps RP, Kaufman J, Blumberg N. Platelet derived CD154 (CD40 ligand) and febrile responses to transfusion. Lancet 2001;357:2023-2024.

16. Danese S, Katz JA, Saibeni S, et al. Activated platelets are the source of elevated levels of soluble CD40 ligand in the circulation of inflammatory bowel disease patients. Gut 2003;52:1435-1441.

17. Kayo S, Ikura Y, Suekane T, et al. Close association between activated platelets and neutrophils in the active phase of ulcerative colitis in humans. Inflamm Bowel Dis 2006;12:727-735.

18. Bessissow T, Lemmens B, Ferrante M, et al. Prognostic value of serologic and histologic markers on clinical relapse in ulcerative colitis patients with mucosal healing. Am J Gastroenterol 2012;107:1684-1692.

19. Bryant RV, Burger DC, Delo J, et al. Beyond endoscopic mucosal healing in UC: histological remission better predicts corticosteroid use and hospitalisation over 6 years of follow-up. Gut 2016;65:408-414.

20. Christensen B, Hanauer SB, Erlich J, et al. Histologic normalization occurs in ulcerative colitis and is associated with improved clinical outcomes. Clin Gastroenterol Hepatol 2017;15:1557-1564.e1.

21. Mooiweer E, Severs M, Schipper ME, et al. Low fecal calprotectin predicts sustained clinical remission in inflammatory bowel disease patients: a plea for deep remission. J Crohns Colitis 2015;9:50-55.

22. Barreiro-de Acosta M, Vallejo N, de la Iglesia D, et al. Evaluation 
of the risk of relapse in ulcerative colitis according to the degree of mucosal healing (Mayo 0 vs 1): a longitudinal cohort study. J Crohns Colitis 2016;10:13-19.

23. Jauregui-Amezaga A, López-Cerón M, Aceituno M, et al. Accuracy of advanced endoscopy and fecal calprotectin for prediction of relapse in ulcerative colitis: a prospective study. Inflamm Bowel Dis 2014;20:1187-1193.

24. Yokoyama K, Kobayashi K, Mukae M, Sada M, Koizumi W. Clinical study of the relation between mucosal healing and long- term outcomes in ulcerative colitis. Gastroenterol Res Pract 2013;2013:192794.

25. Voudoukis E, Karmiris K, Oustamanolakis P, et al. Association between thrombocytosis and iron deficiency anemia in inflammatory bowel disease. Eur J Gastroenterol Hepatol 2013;25:12121216.

26. Kulnigg-Dabsch S, Evstatiev R, Dejaco C, Gasche C. Effect of iron therapy on platelet counts in patients with inflammatory bowel disease-associated anemia. PLoS One 2012;7:e34520. 